\title{
CONFORT CLIMÁTICO, CAMBIO CLIMÁTICO Y ACTIVIDAD TURÍSTICA EN ALICANTE
}

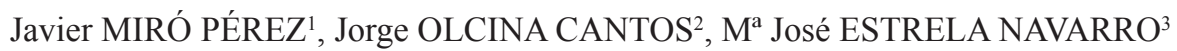 \\ y Vicente CASELLES MIRALLES ${ }^{4}$ \\ ${ }^{1}$ Departamento de Física de la Tierra y Termodinámica. Universitat de València. \\ ${ }^{2}$ Instituto Interuniversitario de Geografia. Universidad de Alicante. \\ ${ }^{3}$ Departamento de Geografia. Universitat de València. \\ ${ }^{4}$ Departamento de Física de la Tierra y Termodinámica. Universitat de València. \\ Javier.Miro-Perez@uv.es
}

\section{RESUMEN}

Los rasgos climáticos son esenciales para comprender la vocación turística de los territorios. En el litoral mediterráneo desde los años sesenta del siglo XX hay una especialización en el producto turístico basado en los rasgos climáticos de verano (sol y playa). La provincia de Alicante es un destino turístico principal en el área mediterránea española y esta actividad representa el 18\% de su PIB provincial. Las condiciones climáticas actuales proporcionan valores de confort climático, según el índice TCI, muy buenos o excelentes durante la temporada alta turística de verano; no obstante, el calentamiento térmico planetario proporciona valores de pérdida de excelencia climática hacia el final del presente siglo en los meses cálidos del año, lo que condicionará la adopción de medidas por parte del sector turístico y de la administración para intentar minimizar el impacto de la nueva realidad climática proyectada.

Palabras clave: clima, confort climático, actividad turística, efectos del calentamiento térmico

\begin{abstract}
Climatic characteristics are essential for understanding the tourist vocation of the territories. In the Mediterranean coast since the sixties of the twentieth century there is a specialization in the tourism product based on summer climate characteristics ("sun and beach"). Alicante province is a premier tourist destination in the Spanish Mediterranean area and this activity represents $18 \%$ of provincial GDP. Current weather conditions provide climate comfort values very good or excellent during peak summer tourist season, according to the TCI index. However, the global warming provides loss in the values of climate excellence towards the end of this century, especially in the warm months of the year, which will determine the action by the tourism sector and public administration to try minimize the impact of the new climate reality.
\end{abstract}

Key words: climate, climate comfort, tourism, effects of global warming

\section{CLIMA Y CONFORT CLIMÁTICO, RECURSO BÁSICO PARA EL TURIS- MO EN ALICANTE. EL EFECTO DEL CALENTAMIENTO GLOBAL}

Los territorios definen su función a partir de unos factores que favorecen el desarrollo de actividades económicas. La existencia de dinamismo social, de capacidad 
emprendedora, las posibilidades de acceso a los recursos naturales, el desarrollo de redes de transporte y comunicación rápidas, la consolidación de mecanismos de financiación o la puesta en marcha de políticas públicas son básicas para entender el mayor o menor éxito de las iniciativas de desarrollo económico. Todos estos aspectos integran el conjunto de factores endógenos y exógenos que, desde el siglo XIX, han permitido la consolidación de las diversas formas de organización económica del espacio geográfico, con sus evidentes efectos socio-territoriales. Y junto a ellos, es pieza básica la presencia de un medio natural con rasgos geológicos, geomorfológicos, climáticos, hídricos, edáficos y biogeográficos favorecedores de las actividades puestas en marcha por las sociedades que en él existen, especialmente en aquellos sectores que dependen ampliamente de estos elementos del medio físico. La actividad turística es una de estas actividades cuyo desarrollo territorial está en estrecha relación con las características físicas del medio donde tiene lugar. La provincia de Alicante, en la fachada mediterránea española, dispone de unos recursos del medio natural muy idóneas para la implantación de actividades de ocio y recreación que son la base del desarrollo turístico.

Entre estos valores del medio, destaca el clima como principal factor de atracción del turismo. La existencia de un clima de filiación mediterránea, con una temperie generalmente tranquila, temperaturas agradables, abundancia de días de sol, soplo de vientos generalmente suaves, efecto refrescante de la brisa en verano, a lo que se une un área de aguas con temperaturas muy agradables para el baño entre la primavera y el otoño, hacen del territorio alicantino un área privilegiada para el desarrollo de la actividad turística. No en vano, desde los años sesenta del siglo pasado, la provincia de Alicante se ha convertido en un destino principal del litoral mediterráneo español y uno de los destacados a escala europea en la modalidad de turismo de sol y playa.

Hay tres procesos principales que pueden desarrollarse en nuestras latitudes mediterráneas por efecto del cambio climático con implicaciones directas en la actividad turística: a) un aumento de los extremos atmosféricos (mayor peligrosidad climática); b) una reducción de precipitaciones y de volúmenes de agua disponible; y c) la pérdida de confort climático en el sur y este peninsular por aumento térmico en los meses centrales de verano.

El V Informe del IPCC (2013-14) señala que el cambio climático que pueda desarrollarse en las próximas décadas, si se sigue confirmando la hipótesis de efecto invernadero como parece, no supondrá sólo el aumento de las temperaturas medias, sino también de su variabilidad. De manera que el clima tiende a un calentamiento, pero también a una mayor frecuencia en el registro de temperaturas extremas, con aumentos en las medias, máximas y mínimas. Al respecto, en el litoral mediterráneo español Quereda et al. (2001) señalan que el aumento de temperaturas medias anuales experimentado desde 1950 en localidades de la fachada este española se debe más al incremento de las mínimas nocturnas que de las máximas extremas diurnas que se hayan podido registrar. En Miró et al. (2006) se señala asimismo que estos cambios son más pronunciados para los meses estivales. De modo que es notable el aumento del número de "noches tropicales" (temperatura mínima $>20^{\circ} \mathrm{C}$ ) que ha tenido lugar en muchas ciudades del litoral mediterráneo español y entre ellas en las ciudades costeras de la provincia de Alicante. En este aumento de las temperaturas mínimas es importante el papel de un creciente efecto urbano proporcionando más persistencia del calor. Pero también es relevante el papel que tiene el calentamiento que experimenta 
el mar Mediterráneo desde hace tres décadas en los meses de verano, tal y como confirman las termografías de satélite (Pastor et al., 2015), unido a un calentamiento continental de base manifestado en las zonas rurales y montañosas, que en este caso es más importante para las temperaturas máximas y partes altas del interior (Miró 2014; Miró et al., 2015, 2016a, 2016b).

En efecto, desde mediados de los años noventa del pasado siglo, la cuenca occidental del Mediterráneo se calienta antes (junio) y más (la temperatura superficial marina llega a alcanzar picos de $27-28^{\circ} \mathrm{C}$ ) de lo que lo hacía en los años ochenta. Este colchón estival de aguas calientes eleva la humedad ambiental, frena el descenso nocturno de la temperatura e incrementa, en suma, la sensación térmica. Y todo ello es ya indicativo de un cambio climático en curso, puesto que el ritmo térmico considerado normal en un espacio geográfico sufre alteraciones, experimenta una pérdida de confort térmico; en suma, el clima cambia.

El informe "Proyecciones climáticas para el siglo XXI" (Aemet, 2015), como actualización de las proyecciones elaboradas en 2011, ha manejado tres variables (temperatura máxima, temperatura mínima y precipitación) en el análisis de la evolución modelizada del clima para el conjunto de España. Para la provincia de Alicante, los datos se pueden estimar a partir de los resultados regionales obtenidos para la Comunidad Valenciana y Murcia. En definitiva, la situación prevista nos habla de un incremento de temperaturas, un aumento de la frecuencia de días cálidos y olas de calor y un descenso preocupante de los valores de precipitación (vid. tabla 1).

\begin{tabular}{|c|c|c|}
\hline & C. VALENCIANA & MURCIA \\
\hline $\begin{array}{l}\mathrm{T}^{\mathrm{a}} \text { máxima } \\
\left({ }^{\circ} \mathrm{C}\right)\end{array}$ & $+1 \mathrm{a}+5$ & $+2 \mathrm{a}+5$ \\
\hline $\begin{array}{l}\text { Duración olas de calor } \\
\text { (días) }\end{array}$ & $5-35$ & $10-45$ \\
\hline $\begin{array}{l}\text { Días cálidos } \\
(\%)\end{array}$ & $15-50$ & $20-55$ \\
\hline $\begin{array}{l}\mathrm{T}^{\mathrm{a}} \text { mínima } \\
\left({ }^{\circ} \mathrm{C}\right)\end{array}$ & $+1 \mathrm{a}+4.5$ & $+2.5 \mathrm{a}+4.5$ \\
\hline $\begin{array}{l}\text { Días de heladas } \\
\text { (días) }\end{array}$ & $-5 a-8$ & -3 a -7 \\
\hline $\begin{array}{c}\text { Noches cálidas } \\
(\%)\end{array}$ & $15-50$ & $20-50$ \\
\hline $\begin{array}{c}\text { Cambio volumen precipitación } \\
(\%)\end{array}$ & $0 \mathrm{a}-10$ & 0 a -5 \\
\hline $\begin{array}{c}\text { Cambio precipitaciones intensas } \\
(\%)\end{array}$ & 0 a -7 & $+1 \mathrm{a}-1$ \\
\hline $\begin{array}{l}\text { Duración períodos secos } \\
\text { (días) }\end{array}$ & $0 a+2$ & $0 \mathrm{a}+2.5$ \\
\hline $\begin{array}{l}\mathrm{N}^{\mathrm{a}} \text { días de lluvia } \\
\text { (días) }\end{array}$ & $-2 a+5$ & $-2.5 a+2.5$ \\
\hline
\end{tabular}

Tabla 1. Cambios en las variables climáticas principales de la Comundiad Valenciana y región de Murcia (horizonte 2100). Fuente: AEMET. Proyecciones climáticas para el s. XXI. Elaboración propia. 
A efectos de confort climático en áreas turísticas del litoral mediterráneo, como la nuestra, resulta significativo el aumento que se prevé en el número de días cálidos, de noches cálidas y en la duración de las olas de calor en todas las regiones del mediterráneo español. Se trata de variables que, unidas a valores de humedad atmosférica altos, se relacionan con la génesis de sensaciones térmicas de disconfort. Y a ello hay que sumar el calentamiento de las aguas del Mediterráneo occidental en los meses cálidos del año que ha experimentado un aumentos en los últimos años prolongando, asimismo, el período de temperaturas elevadas $\left(<25^{\circ} \mathrm{C}\right)$ entre junio y septiembre.

\section{DATOS Y MÉTODOS}

El presente estudio ha llevado a cabo una evaluación a escala de detalle de cambios previstos en el confort climático durante el siglo XXI, en un contexto de cambio climático, a partir de la aplicación del Índice de Confort Climático de Mieczkowski (Mieczkowski, 1985), ó TCI, en la provincia de Alicante. Esta evaluación se ha aplicado para cada estación del año, según el siguiente modo: primavera (marzo, abril y mayo), verano (junio, julio y agosto), otoño (septiembre, octubre y noviembre), e invierno (diciembre, enero y febrero).

Se ha calculado el TCI inicial o de referencia que da cuenta de la situación actual (inicio del siglo XXI) de confort climático para Alicante, a partir de datos observados en los últimos decenios. A continuación se ha aplicado el cambio previsto para calcular la hipotética situación que adoptará el TCI a finales del siglo XXI, y la evaluación del cambio previsto.

La formulación del TCI, datos utilizados, aplicación y proyección futura se desarrollan en los siguientes subapartados.

\section{1. Índice de Confort Climático de Mieczkowski}

En aras de establecer una herramienta que mida la aptitud turística de un clima, la comunidad científica ha desarrollado múltiples índices que combinan variables climáticas tales como las temperaturas, insolación, precipitaciones, humedad, o el viento, de tal manera que se sintetice en un único parámetro dicha aptitud. El quizás más conocido y empleado al respecto es el Índice de Confort Climático de Mieczkowski (Mieczkowski, 1985) ó TCI. Éste se determina por la suma ponderada de 5 subíndices. $\mathrm{Su}$ formulación y forma de resolución es la siguiente:

$$
\mathrm{TCI}=8 \mathrm{CID}+2 \mathrm{CIA}+4 \mathrm{R}+4 \mathrm{~S}+2 \mathrm{~W}
$$

donde CID es el índice de confort diurno, determinado por la temperatura y humedad a la hora de mayor calor (temperatura máxima y humedad mínima diarias medias); CIA es el subíndice de confort medio, determinado por la temperatura y humedad medias; $\mathrm{R}$ es el subíndice pluviométrico determinado por la precipitación total media (mensual, estacional o anual, según resolución temporal de cálculo del TCI); S es la cantidad efectiva media de horas de sol al día; y W es la velocidad media del viento.

Estos subíndices no se calculan a partir de las unidades en que se expresan las variables climatológicas utilizadas, sino que éstas sirven para ponderar un valor den- 
tro de una horquilla fija que va de 0 (desfavorable) a 5 (óptimo) para $\mathrm{R}, \mathrm{S} \mathrm{y} \mathrm{W}$, que en el caso del CID y CIA se establece entre -3 y 5. Ello supone, al aplicar la fórmula anterior, que el valor máximo posible para el TCI es 100 (valor idealmente óptimo). De esta manera, según el TCI resultante, se establece el grado de confort y aptitud turística del clima de acuerdo a la Tabla 1.

\begin{tabular}{|l|l|}
\hline Valor TCI & Categoría \\
\hline $90-100$ & Ideal \\
\hline $80-89,9$ & Excelente \\
\hline $70-79,9$ & Muy bueno \\
\hline $60-69,9$ & Bueno \\
\hline $50-59,9$ & Aceptable \\
\hline $40-49,9$ & Marginal \\
\hline $30-39,9$ & Desfavorable \\
\hline $20-29,9$ & Muy desfavorable \\
\hline $10-19,9$ & Extremadamente desfavorable \\
\hline$<10$ & Imposible \\
\hline
\end{tabular}

Tabla 1: Categorías del TCI (Mieczkowski, 1985).

\subsection{Datos empleados en el cálculo del TCI de referencia}

Para una correcta estimación a fina escala, la variable más decisiva, por ser la que más fluctúa en la vecindad espacial al depender mucho de la altitud, es la temperatura. Por ello se ha hecho uso del dataset SDSITVC (Miró, 2014; Miró et al., 2015, 2016a y 2016b) como fuente de temperaturas a fina escala. Dicho dataset cubre la Comunidad Valenciana y áreas limítrofes con una resolución de $90 \mathrm{~m}$, homogeneidad, y para el periodo completo 1948-2011. Para las precipitaciones se ha hecho uso de todas las series de la red de estaciones pluviométricas de AEMET disponibles en Alicante, con al menos 20 años observados entre 1950 y 2010, con un control de calidad previo.

Sin embargo, el resto de variables necesarias (humedad, insolación y viento) no están disponibles para la red de observatorios de AEMET con más de 20 años observados, a excepción de los escasos disponibles de primer orden, insuficientes para una cartografía de detalle. Por ello, como fuente para estas variables, se ha hecho uso de la red de estaciones automáticas del IVIA-SIAR ${ }^{1}$, utilizando los datos de 20 estaciones, observados entre 1999 y 2012.

Cabe indicar que, para poder realizar el cálculo espacial del TCI fue necesario interpolar previamente cada una de las variables utilizadas según las mismas características del SDSITVC, esto es, una resolución espacial de 90 m (coordenadas UTM huso 30). Se interpolaron mediante kriging ordinario, a excepción de las precipitaciones con las que se usó el método de mínima curvatura.

1 Instituto Valenciano de Investigaciones Agrarias - Sistema de Información Agroclimática para el Regadío (http://eportal.magrama.gob.es/websiar) 
Aunque los periodos de observación no son coincidentes para todas las variables, podemos asumir que permiten una caracterización del TCI lo suficientemente buena para ser representativa de su estado inicial al comienzo del siglo XXI.

\subsection{Proyección del TCI al periodo 2071-2100}

Para el cálculo de la proyección futura del TCI se ha aplicado básicamente el cambio causado por el forzamiento térmico asociado al cambio climático. El cambio previsto en las temperaturas es el que hoy por hoy está relativamente mejor establecido, y también el que, por otro lado, puede mayormente forzar un cambio en el confort climático de la región de estudio. Por el contrario, las proyecciones para precipitaciones arrojan resultados más dispares, al igual que las escasas proyecciones existentes para las otras variables como la humedad, la insolación o el viento (Argüeso, 2011; Brunet et al., 2009; IPCC, 2013; Morata, 2014). Por tanto, para el cálculo de la proyección se ha decidido ponderar únicamente el cambio de confort atribuible a un cambio de temperaturas, dejando por defecto sin cambios el resto de variables.

Para calcular el cambio térmico al periodo 2071-2100 en primer lugar se ha tomado la información a fina escala del SDSITVC sobre patrones espaciales de cambio térmico ya en curso en las últimas décadas, y que han sido bien establecidos en Miró et al. $(2015,2016 \mathrm{a}, 2016 \mathrm{~b})$. A continuación, la magnitud de dichos patrones espaciales se ha proyectado al futuro aplicando el forzamiento térmico previsto por AEMET en su modelo de regionalización AR5-IPCC para el periodo 2071-2100 (http://www. aemet.es/es/serviciosclimaticos/cambio climat). El resultado es una proyección de cambio basada en los patrones espaciales de cambio a fina escala ya detectados actualmente, pero redimensionada según la estimación general de cambio futuro en la región proporcionada por la proyección de AEMET.

\section{RESULTADOS}

Se muestra en primer lugar (Fig. 1) la estimación del TCI de referencia, o sea, en un punto de partida representativo de la actualidad.

Como es de esperar, se parte de una situación en la que es la estación estival la que proporciona mejores niveles en el TCI. Toda la franja litoral y las áreas montañosas prelitorales, dulcificadas por las brisas de origen marino, gozan de un TCI en la categoría de 'excelente' durante el verano. Las áreas interiores que bajan un escalón, a 'muy bueno', coinciden con aquellas en que las temperaturas máximas caniculares se disparan más y la oscilación térmica diurna es mayor.

En el otro extremo, el invierno es la estación con niveles de TCI más bajos, así como se dan las mayores diferencias entre el interior montañoso y el litoral. Con todo, todavía la franja litoral y la mayor parte de la mitad Sur mantienen un nivel en la categoría de 'bueno', que pasa a ser 'aceptable' en la mayor parte del interior Norte. Pero llega a ser 'marginal en la zona más montañosa entre las comarcas de la Marina Alta y El Comtat, y en las cumbres más elevadas.

Las estaciones equinocciales marcan una transición. De ellas, la primavera es la que muestra niveles de confort mejores, cuando alcanza a ser 'excelente' en el litoral del tercio Sur y algún enclave del litoral central, predominando la categoría 'muy bueno' en la mayor parte de la provincia, y la categoría 'bueno' en la zona montañosa del Norte. Mientras, en otoño, seguramente debido a una mayor probabilidad de lluvias, el 
TCI queda en niveles algo inferiores, sobre todo en el tercio Nordeste. Aun así se mantiene la categoría de 'muy bueno' en toda la mitad Sur y tercio Oeste de la provincia.

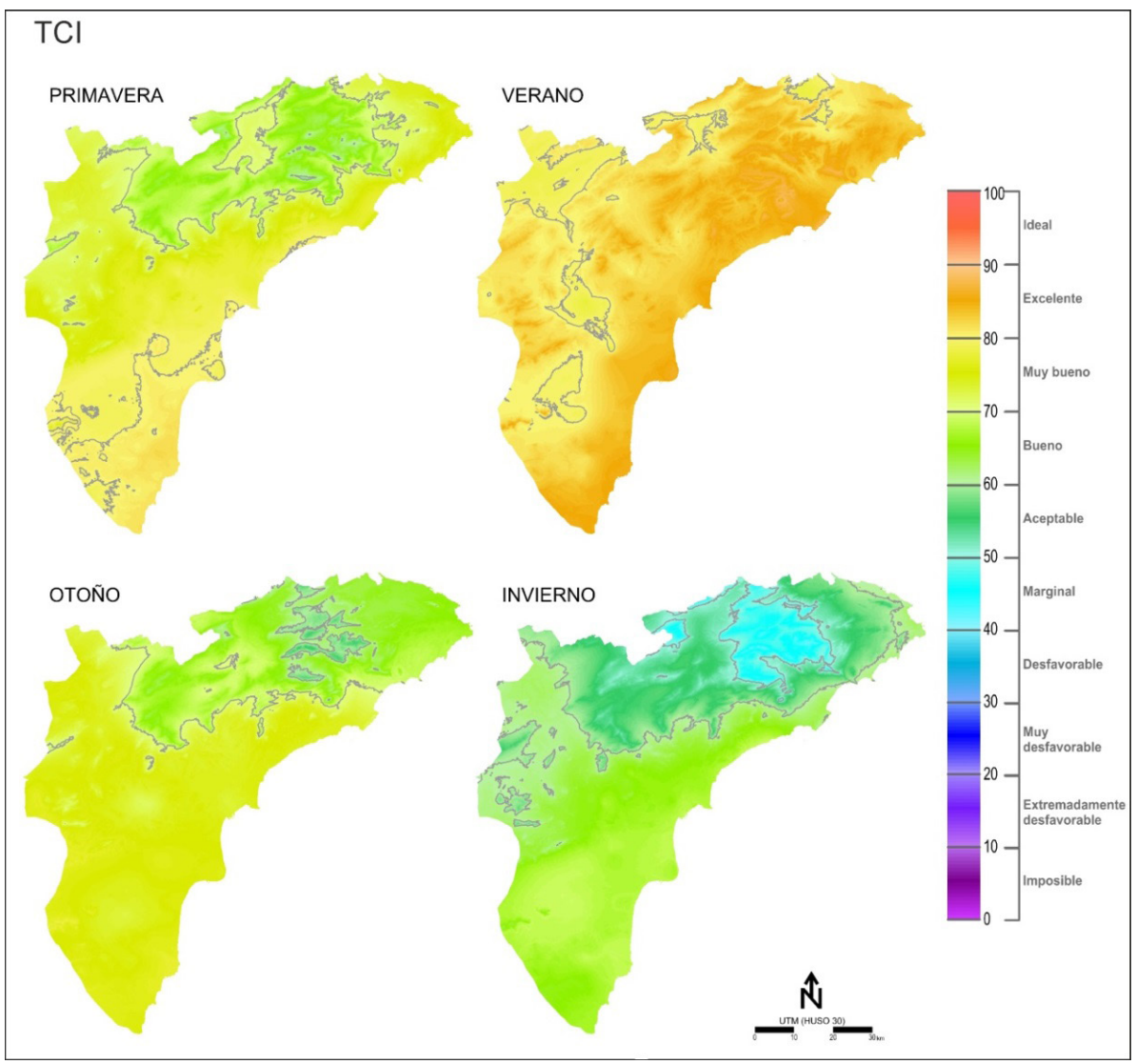

Figura 1. TCI de referencia (actual) calculado para cada estación del año.

Sobre la base del TCI de referencia (Fig. 1), la Figura 2 muestra la magnitud de cambio previsto para el periodo 2071-2100 en unidades propias del TCI.

Puede observarse una pérdida clara de confort para el caso del verano, asociada a unas temperaturas por encima del nivel óptimo de confort térmico. La pérdida es mayor en los valles y hoyas más septentrionales (hasta 7 puntos en algún lugar), pero también es cercana a los 5 puntos de pérdida de confort en la mayor parte del litoral. El descenso sólo es débil, y hasta se invierte en algún caso, para las montañas y partes más elevadas, al menos para las más expuestas a las brisas, que mantendrían aquí su confort debido a la pérdida de temperatura con la altitud.

Sin embargo, el caso contrario ocurre para el invierno. En este caso tenemos una mejora de confort en toda la provincia (entre 2 y 5 puntos), indicando que la pérdida de confort en verano se compensaría con una prolongación de buenos niveles de confort al resto del año. 


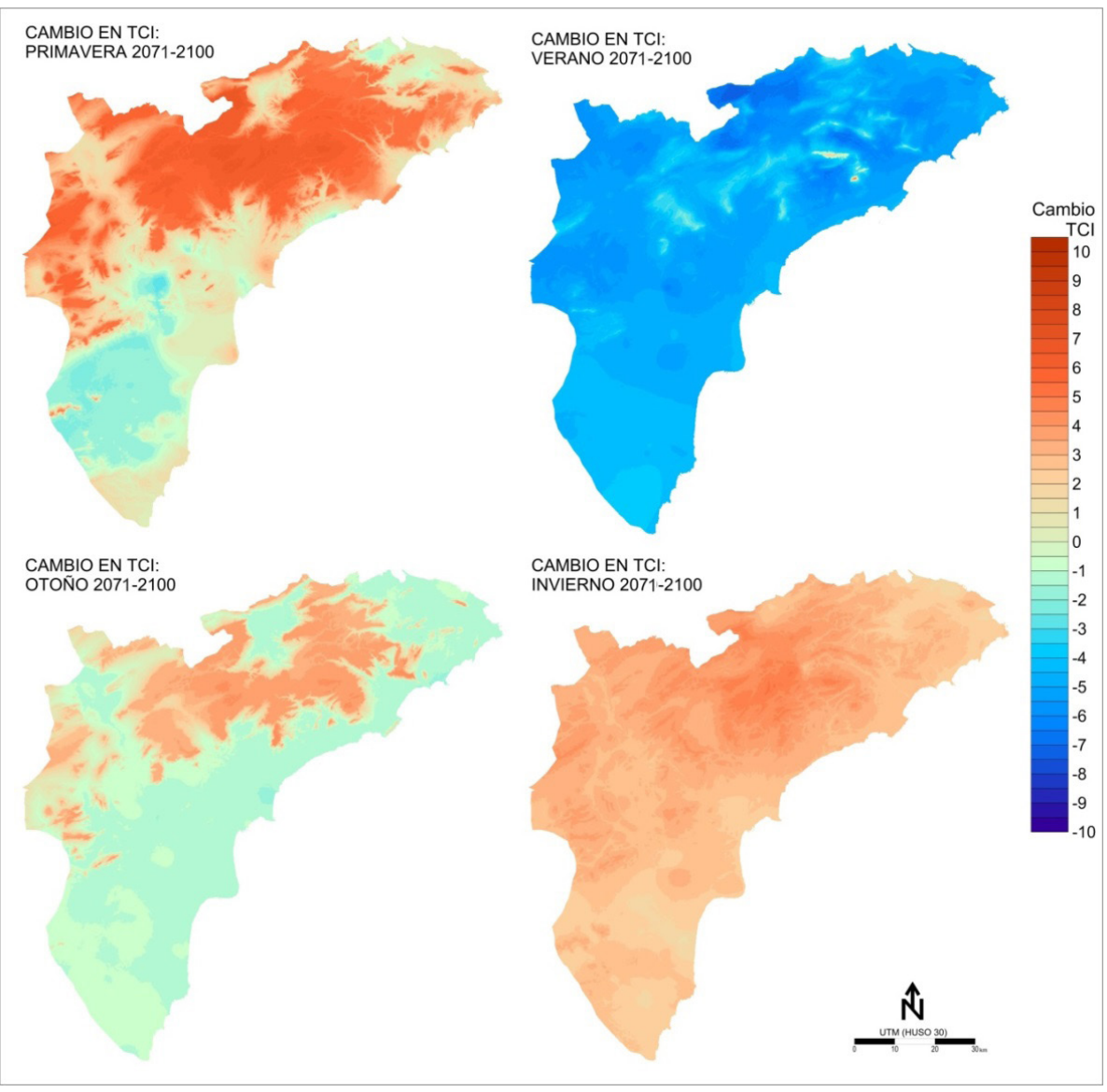

Figura 2. Cambio previsto en unidades del TCI para el periodo 2071-2100 con respecto al TCI de referencia, según la proyección climática utilizada en el estudio.

Es muy destacable que los mayores aumentos de confort se esperen para las áreas montañosas y partes altas, lo cual se aprecia especialmente en las estaciones equinocciales, en las que aparecen tendencias de cambio opuestas entre las tierras altas (aumento de confort) y las tierras bajas (sin cambios o muy ligero descenso). La diferencia es más acusada en primavera, cuando la proyección señala un acusado aumento de confort ( 5 a 7 puntos en TCI) en las partes altas por encima de 500mts, mayor cuanto más elevadas. Por el contrario en el litoral los cambios son menos importantes, e incluso aparece una débil perdida de confort hacia el interior de la comarca de la Vega Baja, donde los episodios de calor ya tendrían en primavera un impacto negativo.

En otoño también se proyecta un aumento de confort en las tierras altas del Norte de la provincia, pero mucho más moderado que en primavera, mientras que la mayor parte del territorio provincial (valles, hoyas y litoral) quedan con cambios muy débiles (pérdida en torno a 1 punto). 


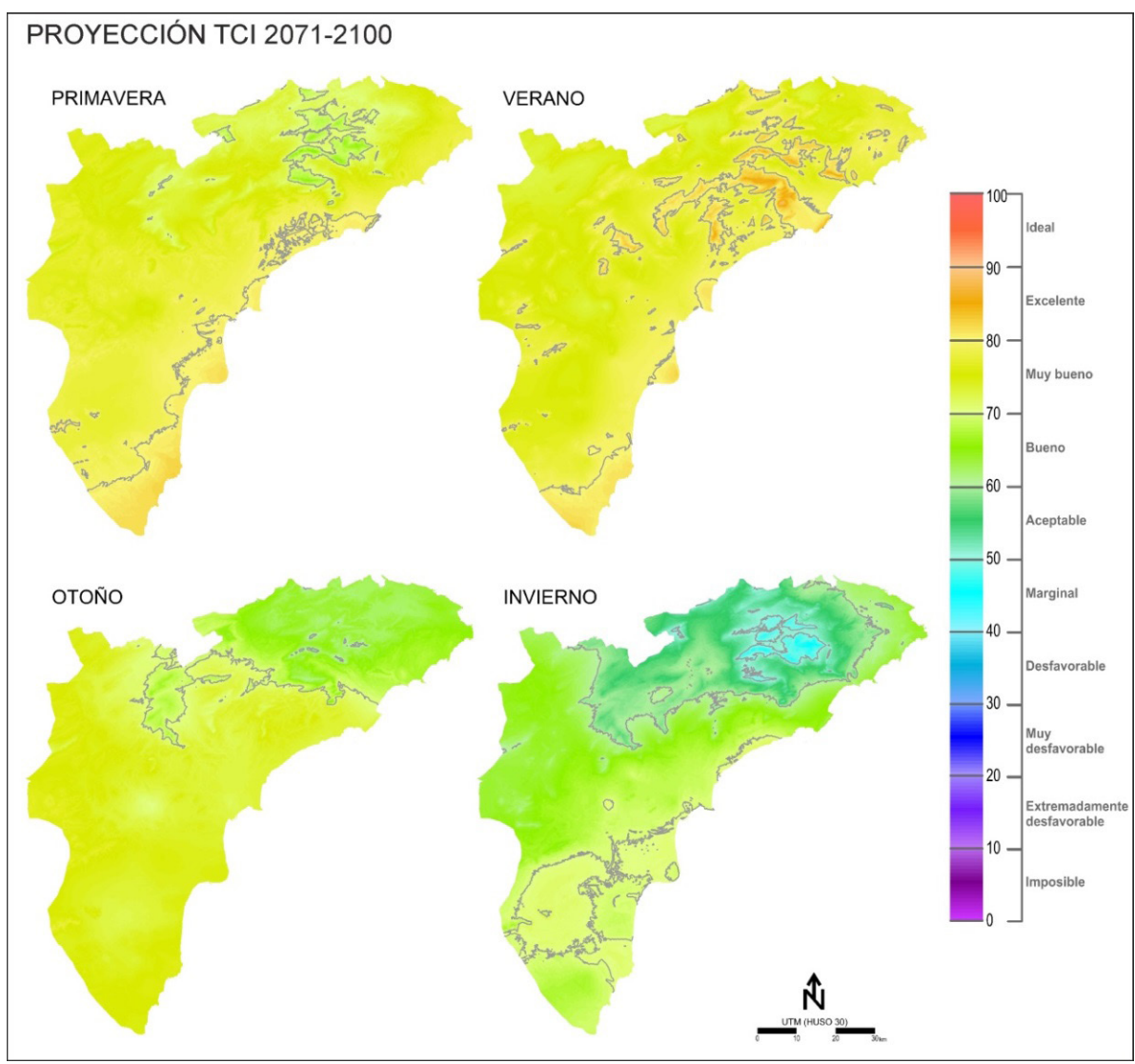

Figura 3. TCI proyectado al periodo 2071-2100 para cada estación del año.

Este panorama proyectado coloca en escena una posible pérdida de confort para el turismo litoral de sol y playa del semestre estival; pero también un considerable aumento de las aptitudes turísticas de la zona montañosa de la provincia a lo largo del año (turismo de espacios naturales, culturales, ocio, etc.), así como mejora las aptitudes del litoral en invierno. No obstante cabe destacar el tercio Sur, en especial el interior de la Vega Baja, como el área en que se produciría una pérdida de confort durante casi todo el año, a excepción sólo del invierno.

Al aplicar los cambios proyectados, el TCI y sus categorías quedarían para el periodo 2071-2100 del modo representado en la Figura 3. Si la comparamos con la Figura 1 se advierten bien los cambios proyectados. Así se observa que, dada una relativa pérdida de confort en verano y un aumento en zonas interiores para la primavera, ahora tanto primavera como verano quedarán prácticamente a la par como las estaciones con mejor aptitud turística, predominando en general un TCI en la categoría de muy bueno.

Al analizar cómo quedan las categorías del TCI proyectado, se destaca en primer lugar la pérdida de la categoría 'excelente' para la mayor parte del territorio en verano. 
Sólo se mantiene en puntos del litoral y las montañas prelitorales cercanas al litoral central. Pero de cara a la primavera esa misma categoría 'excelente' aumenta algo su presencia en el litoral central y Sur, y casi todo el sector montañoso del Norte de Alicante asciende un escalón, de 'Bueno' a 'Muy Bueno'.

En otoño se aprecian también mejoras en la categoría del TCI hacia el Nordeste de la provincia, extendiéndose más la categoría de 'Muy bueno', y casi desapareciendo la categoría 'Aceptable' en las zonas altas, por la de 'Bueno'. El resto de la provincia se mantendría en la categoría de 'Muy bueno'.

Y en invierno se destaca bastante la mejora del TCI en el interior montañoso al Norte, de manera que los sectores bajo categoría 'Marginal' se reducen hasta sólo quedar enclaves aislados. Pero también se destaca la aparición de la categoría 'Muy bueno', que antes no llegaba a estar presente en invierno, hacia el litoral central y Sur de la provincia.

\section{CONCLUSIONES Y RECOMENDACIONES}

El impacto de estos procesos de cambio climático afectará previsiblemente a los niveles de confort climático y la propia aptitud del clima para el turismo. Sin embargo, estos cambios podrían ser positivos o negativos, dependiendo de la época del año. La proyección realizada del índice de confort climático hacia el final del presente siglo presenta una pérdida clara de confort para el caso del verano, esencialmente a causa de un aumento de episodios de calor asociados a bajo confort. Sin embargo, el caso contrario ocurre para el invierno. En este caso tenemos una mejora de confort en toda la provincia, como se ha señalado, lo que es indicativo de que, aunque se pronostique una pérdida de confort en verano, se compensaría con una prolongación de buenos niveles de confort al resto del año. Esta mejoría en confort es más evidente en las zonas montañosas que originalmente tenían niveles de confort más bajos fuera del verano.

A efectos de planificación y adaptación de los espacios turísticos del litoral mediterráneo a los efectos del cambio climático, estos datos de proyección futura de variables atmosféricas, índices de confort y de temperatura de aguas marinas suponen:

- La posibilidad de modificar el calendario de "temporada alta", muy centrada en la actualidad en julio y sobre todo agosto, que podrá prolongarse desde junio a septiembre, incluidos, además de considerar la estación primaveral junto con octubre muy aptos para las estancias turísticas en este espacio geográfico. Junto a ello, ganarían interés actividades turísticas en áreas montañosas de mayor altitud en la provincia.

- La necesidad de acondicionamiento climático de los establecimientos turísticos, de las viviendas residenciales y de las tramas urbanas a una situación más habitual de altas temperaturas y elevada humedad, diurna y nocturna, a los efectos de compensar el disconfort térmico que se estima creciente en los espacios costeros, especialmente a partir de mediados del siglo actual.

- La obligación de tener bien diseñados los sistemas de abastecimiento de agua, a escala regional y local, en un área con natural escasez de recursos, donde la propia reducción de precipitaciones, el aumento de la irregularidad en su desarrollo y el 
incremento de la evaporación en embalses, originará una disminución de volúmenes de agua superficial disponible.

- La necesidad, asimismo, de modificar, en la escala local, los protocolos de protección civil y sanidad pública, puesto que se van a alterar los calendarios de riesgo frente a determinados peligros de causa climática (tormentas y lluvias intensas por la presencia de aguas calidad en el Mediterráneo occidental durante un período del año mayor), así como la frecuencia e intensidad de aparición de extremos atmosféricos (olas de calor y sus efectos en grupos de riesgo). En esta cuestión será necesario mejorar los sistemas de drenaje de precipitaciones intensas en las ciudades turísticas en aras a la reducción de sectores de riesgo de anegamiento e inundación.

\section{AGRADECIMIENTOS}

Este trabajo se ha podido realizar gracias al programa para grupos de investigación de excelencia PROMETEOII (2014/086) de la Comunidad Valenciana, y el proyecto CGL2015- 64268-R (MINECO/FEDER, UE) del Ministerio de Economía y Competitividad y el FEDER.

\section{REFERENCIAS}

Argüeso, D. (2011). High-resolution projections of climate change over the Iberian Peninsula using a mesoscale model. Ph. D. Thesis. Departamento de Física Aplicada. Universidad de Granada.

Brunet, M., Casado, M.J., De-Castro, M., Galán, P., López, J.A., Martín, J.M., Pastor, A., Petisco, E., Ramos, P., Ribalaygua, J., Rodríguez, E., Sanz, I. y Torres, L. (2009). Generación de escenarios climáticos regionalizados para España. Agencia Estatal de Meteorología. Madrid, 157 p.

IPCC (2013). Climate Change 2013: The Physical Science Basis. Contribution of Working Group I to the Fifth Assessment Report of the Intergovernmental Panel on Climate Change [Stocker, T.F., D. Qin, G.-K. Plattner, M. Tignor, S.K. Allen, J. Boschung, A. Nauels, Y. Xia, V. Bex and P.M. Midgley (eds.)]. Cambridge University Press, Cambridge. United Kingdom and New York, NY, USA, 1535 pp, doi: 10.1017/CBO9781107415324

Mieczkowski, Z. (1985). The tourism climatic index: a method of evaluating world climates for tourism. The Canadian Geographer, 29: 220-33

Miró, J.J., Estrela, M.J., Millán, M. (2006). Summer Temperature Trends in a Mediterranean Area (Valencia Region). International Journal of Climatology, 26, 1051-1073, doi: 10.1002/joc.1297

Miró J.J. (2014). Downscaling estadístico de series climáticas mediante redes neuronales: Reconstrucción en alta resolución de la temperatura diaria para la Comunidad Valenciana. Interpolación espacial y análisis de tendencias (19482011). Tesis Doctoral. Universidad de Alicante, Fundación Centro de Estudios Ambientales del Mediterráneo, y Universidad de Valencia. 523p., doi: 10.13140/ RG.2.1.2059.1523 
Miró, J.J., Estrela, M.J. y Olcina-Cantos, J. (2015). Statistical downscaling and attribution of air temperature change patterns in the Valencia region (1948-2011). Atmos. Res., 156: 189-212, doi:10.1016/j.atmosres.2015.01.003

Miró, J.J., Estrela, M.J. y Olcina-Cantos, J. (2016a). Reconstrucción de la señal térmica local en la Comunidad Valenciana entre 1948 y 2011 a partir de un downscaling estadístico mediante una red neuronal artificial: Detección de patrones locales de cambio. Boletín de la Asociación de Geógrafos Españoles, 70, 113-147 (eng:471475), doi:10.21138/bage. 2165

Miró, J.J., Estrela, M.J., Caselles, V. y Olcina-Cantos, J. (2016b). Fine-scale estimations of bioclimatic change in the Valencia region, Spain. Atmos. Res., 180: 150-164, doi:10.1016/j.atmosres.2016.05.020

Morata, A. (2014). Guía de escenarios regionalizados de cambio climático sobre España a partir de los resultados del IPCC-AR4. Nota técnica AEMET. 197p.

Olcina Cantos, J. y Miró Pérez J.J. (2016). "El clima, recurso básico del turismo alicantino. De la valoración del clima invernal al aprovechamiento intensivo del clima estival", Rev. Canelobre, $\mathrm{n}^{\circ} 65$ (monográfico sobre la actividad turística en la provincia de Alicante) Diputación Provincial de Alicante, 3-21.

Pastor, F., Valiente, J.A. y Estrela, Ma J. (2015). Sea surface temperature and torrential rains in the Valencia region: modelling the role of recharge areas. Natural Hazards and Earth System Sciences, $n^{\circ}$ 15, p.1677-1693, doi:10.5194/ nhess-15-1677-2015.

Quereda Sala, J. et al. (2001). Nuestro porvenir climático, ¿Un escenario de aridez?. Universitat Jaume I, Castellón, 224 p.

Vera Rebollo, J. F. (1985). "Las condiciones climáticas y marítimas como factores de localización del turismo histórico alicantino", Investigaciones Geográficas $\mathrm{n}^{0} 3$, 161-178.

Vera Rebollo, J.F. (1987). Turismo y urbanización en el litoral alicantino. Instituto de estudios Juan Gil Albert, Diputación Provincial de Alicante. 